\title{
Éléments pour une faune des Siphonaptères du Burundi :
}

\author{
Étude de la zone de Tora
}

\section{par C. GUIGUEN (*), J. VISSAULT (**) et J.-C. BEAUCOURNU (*)}

* Laboratoire de Parasitologie (Entomologie médicale), Faculté de Médecine, F 35043 Rennes Cedex.

** Chargé d'études par le F.E.D. des "moyens de lutte contre les Rongeurs du Theier», B.P. 103, Bujumbura (Burundi).

\section{Résumé.}

Des prélèvements effectués à Tora (Sud du Burundi) sur 318 rongeurs ont livré 614 siphonaptères appartenant à 14 espèces ; les plus abondantes sont Dinopsyllus longifrons, D. apistus et Hypsophthalmus campestris. Les auteurs notent le haut indice pulicidien des rongeurshôtes.

Compte tenu des puces notées (Xenopsylla spp. et Pulex se rencontrent dans les habitations), des hôtes et de divers facteurs abiotiques, le risque pesteux est indéniable dans la région étudiée.

\section{Summary.}

Some elements for a fauna of the Siphonaptera of Burundi : a study of the Tora area.

614 siphonaptera belonging to 14 species have been collected on 318 rodents in Tora (South of Burundi); the most abundant are Dinopsyllus longifrons, D. apistus and Hypsophthalmus campestris. The authors notice the great pulicidian index of the host-rodents.

On account on the noticed fleas (Xenopsylla spp. and Pulex are present in the habitations), hosts and various abiotical factors, the plague risk is undeniable in the studied area.

Accepté le 29 octobre 1979. 


\section{I. - Introduction}

Le Burundi, pays du Centre Est africain (situé entre $2^{\circ} 30^{\prime}$ et $4^{\circ} 28^{\prime}$ de latitude Sud et entre $29^{\circ}$ et $30^{\circ} 55^{\prime}$ de longitude Est) d'une superficie de $27834 \mathrm{~km}^{2}$, possède une des plus fortes densités humaines d'Afrique : 140 habitants au $\mathrm{km}^{2}$.

Bien que situé près de l'Equateur, ce pays jouit d'un climat privilégié, ses « mille et une collines » s'étageant du Tanganyika (770 m d'altitude) au Mont Téza (2 $665 \mathrm{~m})$. Les vallées bénéficient des vents frais des collines, tandis que la grande plaine de l'Imbo subit l'influence modératrice du Lac.

Essentiellement enserré entre la Tanzanie à l'Est et le Zaïre à l'Ouest où règne une notable endémie pesteuse, il nous paraît à craindre que le Burundi ne puisse échapper indéfiniment à cette zoonose. C'est dans cette optique que nous avions entrepris ce travail.

Par suite d'éléments indépendants de notre volonté, un seul gîte a pu être étudié et encore n'a-t-il pu être suivi autant que nous l'aurions souhaité. Toutefois, aucun autre sondage n'ayant, à notre connaissance, été effectué dans ce pays, nous présentons ici les éléments que nous avons pu réunir sur les Siphonaptères du Burundi.

\section{If. - Biotope, Méthodes, Hôtes}

Pendant une période de sept mois (décembre 1977-juin 1978), deux d'entre nous (C. G. et J. V.) ont capturé des rongeurs et en ont récolté les puces (Siphonaptera) à Tora. Cette station est située dans le périmètre de l'usine théicole, dans la province de Bururi, à environ $40 \mathrm{~km}$ au S.-S.E. de Bujumbura, la capitale. C'est une zone de collines d'élevages et de cultures, "collines " atteignant toutefois avec le Donge l'altitude de $2460 \mathrm{~m}$, l'altitude moyenne étant de l'ordre de $2000 \mathrm{~m}$. Autrefois recouverte de bambou, il ne reste dans cette zone que de faibles vestiges des forêts primaires: les seuls couverts notables actuellement sont constitués d'essences d'importation, Eucalyptus spp. en particulier.

En relation avec l'altitude, le climat se caractérise par de notables amplitudes thermiques nycthémérales; les gelées sont courantes pendant la nuit, alors que les températures maximales sont supérieures à $20^{\circ}$ toute l'année.

On note les quatre "saisons » classiques en Afrique équatoriale qui, au Burundi, se répartissent ainsi : une petite saison sèche en janvier, une petite saison des pluies de février à la mi-mai, une grande saison sèche de la mi-mai à la mi-août, et une grande saison des pluies de la mi-août à décembre inclus (fig. 1).

Localisée dans le temps, notre enquête l'a été également dans l'espace. Tous nos piégeages ont été effectués sur une superficie de 200 ha environ. En dehors de quelques prélèvements effectués autour des habitations indigènes, les Rugos, et 
dans les haies vives qui les entourent, tous les autres proviennent de biotopes ouverts, cultivés ou l'ayant été : champs de maïs, prairies et essentiellement friches.

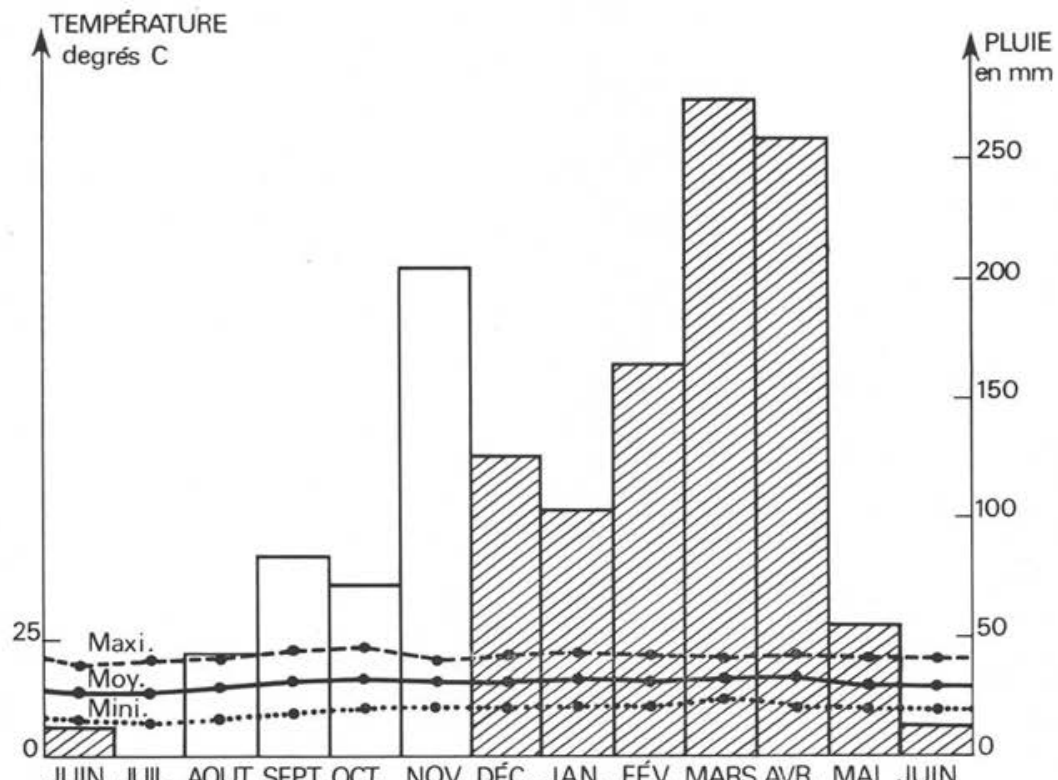

Fig. 1. Régime mensuel des pluies et températures de juin 1977 à juin 1978 (Période de piégeage en hachuré).

Trois cent dix-huit rongeurs (1) appartenant à 12 genres et à 2 familles (Muridae et Cricetidae) ont été capturés à l'aide de trappes en grillage, à porte retombante. Nous en donnons ci-dessous le décompte (tableau I). Relevons d'emblée que 5 espèces (Oenomys hypoxanthus, Lophuromys sikapusi, Otomys denti, Mastomys natalensis et Dasymys incomtus) regroupent $92 \%$ des hôtes capturés, Oenomys en représentant $25 \%$ à elle seule. Par contre, Lophuromys (20\% des captures seulement) a fourni, qualitativement et quantitaivement, le plus de puces: 12 espèces sur $14 ; 30,6 \%$ du total des prises viennent de ce rongeur, soit un indice pulicidien de 2,98.

Il faut à ce propos souligner le niveau relativement élevé de l'indice pulicidien des rongeurs rencontrés ici. Sur l'ensemble des captures il est de 1,93; si l'on ne considère que les 5 genres régulièrement présents (2), l'indice varie de 1,31 à 2,98 ! Mas-

(1) La classification suivie est celle de Delany (1975).

(2) Rattus est abondant autour des Rugos. Nous n'y avons piégé que quelques jours, ce qui explique le peu de captures opérées. 
tomys, dont le rôle épidémiologique est bien connu (Davis, 1953 ; Gratz et Arata, $1975 \ldots$... est en seconde position avec 2,03 (tableau I). Nous estimons d'ailleurs que ces indices sont minorés non seulement par le type de piège utilisé ici, mais aussi par les conditions de piégeage (les rongeurs capturés vivants n'étant le plus souvent étudiés que 2 à 3 jours après la prise !). Or, rappelons que Van Riel et Mol (1939) étudiant le foyer pesteux, actif, du Kivu (ou foyer du lac Edward), ne trouvaient qu'un indice pulicidien de 0,55 !

Sur le plan écologique, Rattus rattus est presque exclusivement synanthrope : Mastomys, comme il est de règle en biotopes ouverts, est tout autant synanthrope que selvatique (Misonne, 1959). Les autres genres sont à considérer comme selvatiques.

Il nous paraît intéressant de signaler que, dans le cadre d'un vaste travail effectué par l'un de nous dans cette même région (J. V.), plus de 7500 Tachyoryctes splendens (Ruppel, 1835) (Rodentia, Rhizomyidae) et environ 25 de leurs nids ont été étudiés : aucun Siphonaptère n'a été vu! Ceci est d'autant plus surprenant que dans des zones relativement voisines d'Afrique, comme le Kenya et la Tanzanie, ce rongeur héberge une faune de puces, riche et spécifique (cf. en particulier Smit, 1960 ; $1975 ; 1976)$.

\section{III. - Les Siphonaptères}

Près de 700 Siphonaptères appartenant à 16 espèces ont été collectés à Tora: deux d'entre elles, Tunga penetrans (L.) et Pulex irritans (L.) sont parasites de l'homme et de certains animaux domestiques. Nous ne faisons donc ici que rappeler leur présence, le problème posé par Pulex ayant été évoqué par ailleurs (Guiguen et Beaucournu, sous presse). Si l'on ne considère que la faune liée aux rongeurs, nos relevés donnent 14 espèces regroupant 614 exemplaires. Il est bien évident que le fait d'avoir prospecté une seule station ne présentant qu'un type de biotope a considérablement réduit l'éventail des captures possibles, un bon nombre d'hôtes, ou même de puces, ne pouvant se rencontrer, par exemple, qu'en biotopes forestiers. Notons que toutes les puces citées ici, à l'exception de $T$. penetrans et Ctenecephalides felis, sont, à notre connaissance, nouvelles pour le Burundi (3).

\section{1. - Liste des espèces récoltées.}

Famille Hystrichopsillidae :

1 - Dinopsyllus (Dinopsyllus) apistus Jordan et Rothschild, 1913.

2 - D. (D.) echinus Jordan et Rothschild, 1913.

3 - D. (D.) longifrons Jordan et Rothschild, 1913.

(3) Lagaropsylla idae, Smit 1957, a été capturé sur Tadarida sp. à Bujumbura. 
4 - Ctenophthalmus (Ethioctenophthalmus) calceatus cabirus Jordan et Rothschild, 1913.

$5-C$. (E.) evidens evidens Jordan, 1929.

$6-$ C. (E.) eximius Jordan et Rothschild, 1913.

7 - C. (E.) phyris Jordan, 1941.

Famille Chimaeropsyllidae (= Hypsophtalmidae):

8 - Hypsophthalmus campestris Jordan et Rothschild, 1913.

Famille Xiphiopsyllidae :

9 - Xiphiopsylla hyparetes Jordan et Rothschild, 1913.

$10-X$. levis Smit, 1960.

Famille Ceratophyllidae:

11 - Nosopsyllus (Nosopsyllus) incisus (Jordan et Rothschild, 1913). Famille Pulicidae :

12 - Ctenocephalides felis strongylus (Jordan, 1925).

13 - Xenopsylla brasiliensis (Baker, 1904).

$14-X$. cheopis (Rothschild, 1903).

\section{2. - Etude systématique.}

Dinopsyllus apistus J. et R., 1913.

Cette puce, décrite de zones d'altitude du Kenya et d'Ouganda, a été retrouvée au Zaïre. Elle est en particulier très abondante dans la région du lac Kivu (Beaucournu et Rahm, 1978), soit à 250 km au N.-N.O., où elle parasite essentiellement Praomys, rongeur rare dans la zone de Tora. Son hôte électif est, ici, Mastomys natalensis (59 exemplaires sur 52 hôtes capturés).

Dans nos relevés, D. apistus représente 19,4\% des puces collectées et n'est surclassé que par Dinopsyllus longifrons.

Ainsi que l'avaient déjà relevé Beaucournu et Rahm (op. cit.), le sex-ratio de cette espìce, est en faveur des mâles, ce qui est relativement rare chez les Siphonaptères. Nous le trouvons ici de 1,83 sur 119 exemplaires (1,44 sur 183 in B. et R.).

Dinopsyllus echinus J. et R., 1913.

La répartition connue de $D$. echinus, également puce altitudinale, comprend le Kenya, l'Ouganda, le Zaïre oriental, le nord de la Tanzanie. Elle est parasite de divers muridés et de Tachyoryctes.

Dans la région du lac Kivu où elle semble rare (op. cit.), son hôte est Lophuromys aquilus. C'est Lophuromys sikapusi que nous trouvons à Tora, où il livre $68 \%$ des exemplaires.

Bien que l'on ne puisse conclure sur des chiffres aussi faibles, le sex-ratio de $D$. echinus semble plus "classique » avec 21 mâles pour 28 femelles. 


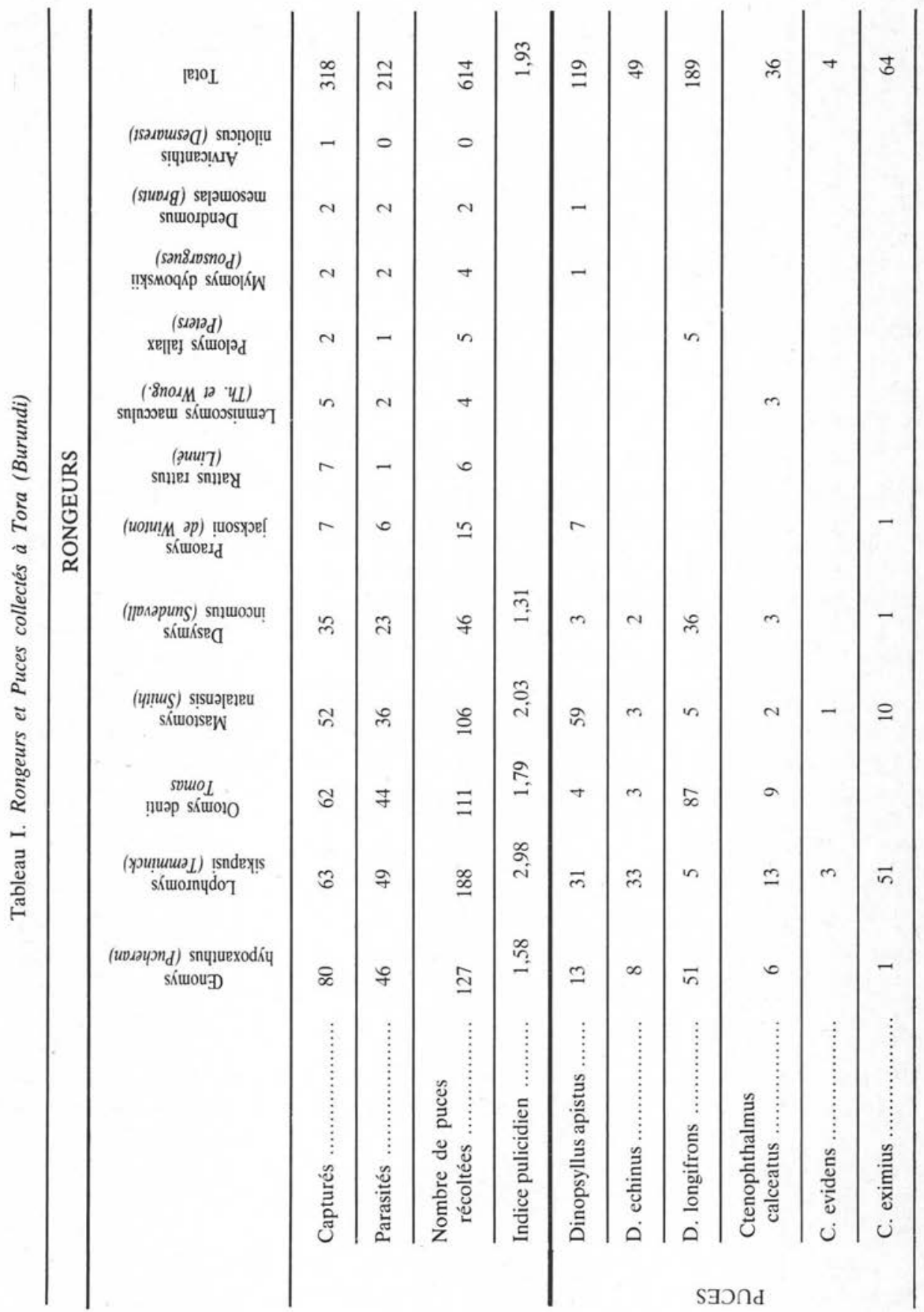




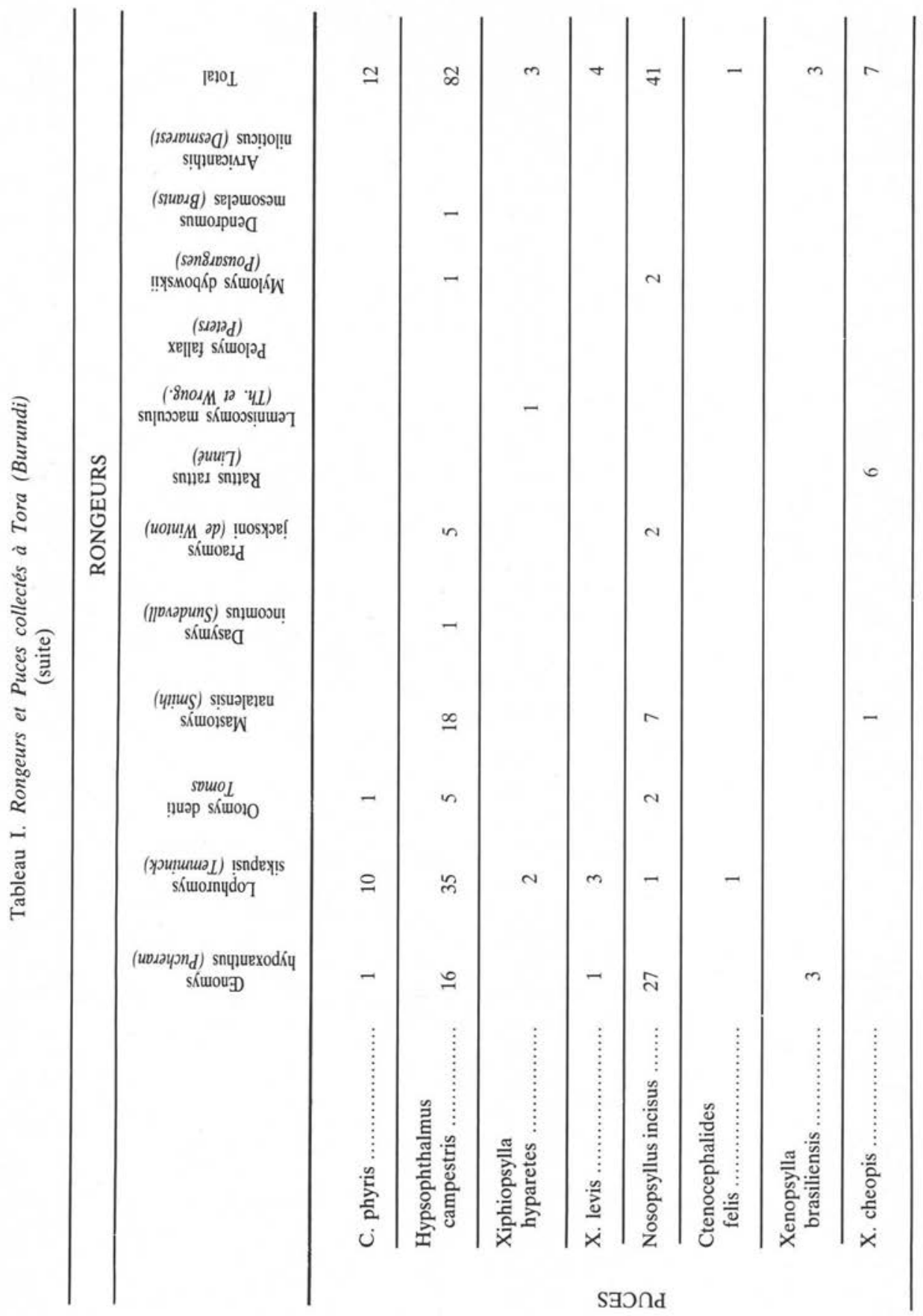


Dinopsyllus longifrons, J. et R., 1913.

D. longifrons a les mêmes exigences écologiques que les précédentes, mais a une répartition plus vaste, puisqu'en plus des pays cités plus haut, on la connaît également du Malawi et de la Zambie selon Haeselbarth (1966), mais Smit (1975) ne retient pas ces deux derniers pays dans sa répartition.

C'est généralement une espèce commune sur bon nombre de muridés et sur Tachyoryctes.

A Tora, D. longifrons est la plus abondante des puces récoltées (189 exemplaires). Ses hôtes électifs sont ici Otomys (Cricetidae), Oenomys et à un moindre degré Dasymys (Muridae). Il est intéressant de noter que Lophuromys abondamment parasité par $D$. echinus et $D$. apistus n'a livré que $5 \mathrm{D}$. longifrons.

Pour autant que nous le sachions, le sex-ratio de cette espèce n'était pas connu. Nos récoltes donnent S.R. $=0,89$.

Ctenophthalmus calceatus cabirus, J. et R., 1913.

Ce Ctenophthalmus est, sans doute, le plus largement répandu des représentants éthiopiens de ce genre et sa répartition recouvre celle de D. longifrons, Zambie et Malawi inclus. Pour Hopkins (1947) sa répartition altitudinale va de 900 à $2400 \mathrm{~m}$.

Comme beaucoup de Ctenophthalmus, il semble assez peu spécifique, les muridés étant ses hôtes classiques.

A Tora, C. calceatus est peu abondant (36 captures) mais son hôte électif semble être Lophuromys, ce qui confirmerait les observations de Beaucournu et Rahm (op. cit.) dans la région du lac Kivu.

Ctenophthalmus evidens evidens, J., 1929.

C. evidens est actuellement représenté par 5 sous-espèces. La forme nominale était seulement connue le long du "Graben » Centre African depuis Blukwa (Zaïre) au nord, à Bufundi (Ouganda). Nous la signalons de Tora, ce qui double approximativement sa zone de répartition, sans modifier son appartenance biogéographique. Notons toutefois qu'elle ne figure pas dans la faune de la région occidentale du lac Kivu étudiée par Beaucournu et Rahm (1978). Les autres sous-espèces sont toutes à distance et de l'autre côté du Graben. Il s'agit, du nord au sud, de evidens elgonensis Jordan 1938 (Mont Elgon, Ouganda), evidens convexus Smit 1975 (Monts Cherangani, Kenya), evidens modicus Jordan 1933 (Nakuru et environs, Kenya) et evidens wilkesi Hubbard 1963 (Cratère du Ngorongoro, Tanzanie et Mont Elgon, Kenya).

Le seul dessin relativement récent de C. e. evidens est celui de Smit (1963). Celui-ci toutefois ne montre pas les sclérifications internes du basimère. Nous redonnons donc ici le tergite IX de deux de nos exemplaires afin de montrer la variabilité de cette sous-espèce (fig. 2).

C. evidens montre une préférence certaine pour Lophuromys, dans la littérature comme dans nos quelques captures. C'est une espèce altitudinale, jamais récoltée jusqu’à présent au-dessous de $1500 \mathrm{~m}$ et généralement vers $2500 \mathrm{~m}$.

Nous en avons récolté 4 exemplaires seulement, 3 mâles et 1 femelle. 


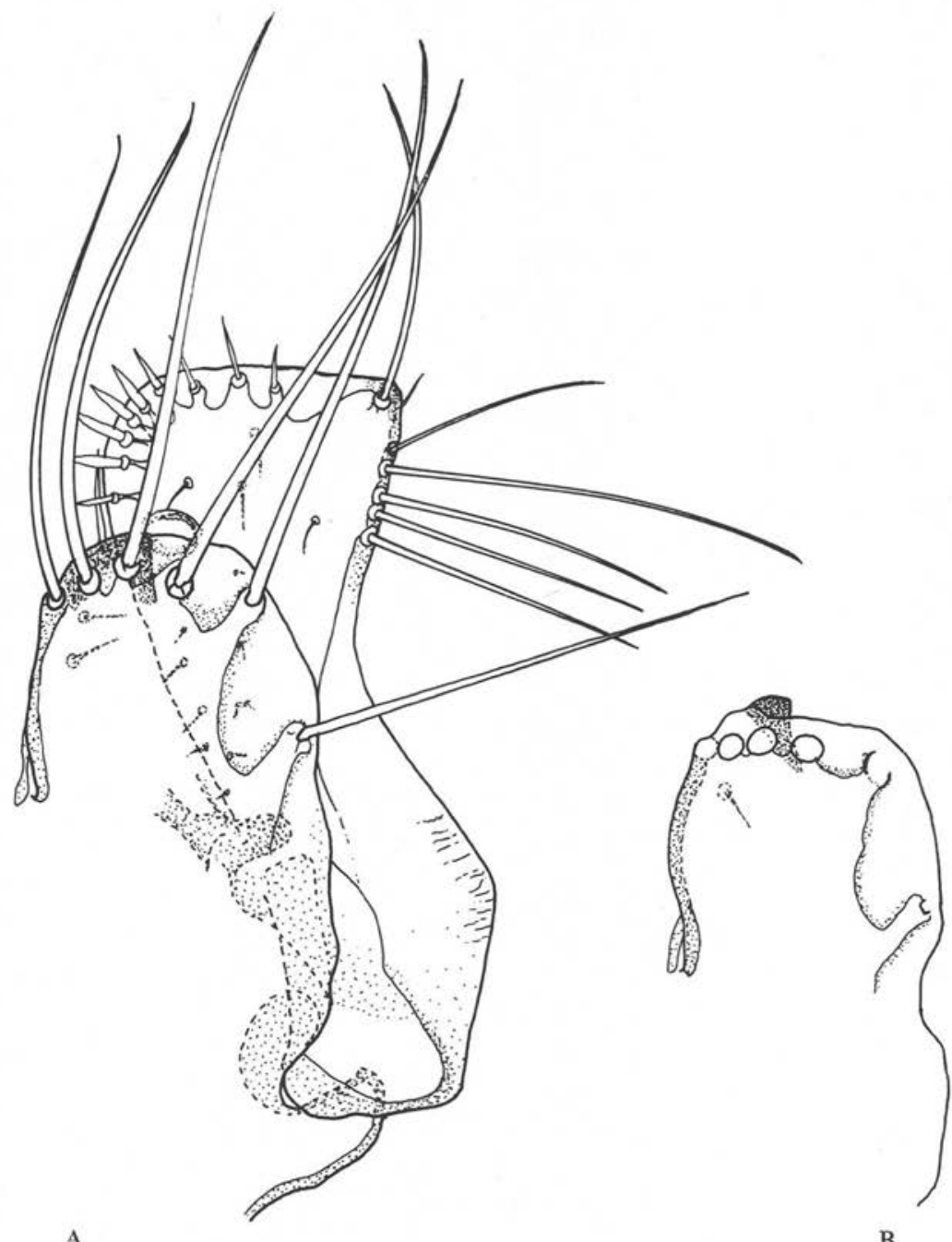

A

Fig. 2. Ctenophthalmus (E.) evidens evidens Jordan 1929, exemplaires de Tora ; $\mathrm{A}$ : basimère et télomère ; $\mathrm{B}$ : basimère.

Ctenophthalmus eximius J. et R. 1913.

Cette espèce est connue du Kenya, de l'Ouganda, du Zaïre oriental, de la Tanzanie, du Malawi et de la Zambie. C'est un parasite de Lophuromys, vivant à moyenne et haute altitude (Haeselbarth, 1966; Smit, 1975; Beaucournu et Rahm, 1978). 
A Tora, C. eximius est relativement abondant (64 exemplaires). Son hôte primaire est Lophuromys qui regroupe $80 \%$ des captures.

Le sex-ratio de cette espèce était, calculé sur 162 exemplaires du Kivu (op. cit.), de 0,7 . Ici sur 64 individus seulement il est de 0,88 , ce qui semble toutefois confirmer un pourcentage « classique ».

Ctenophthalmus phyris Jordan 1941.

Jusqu'à ce travail, $C$. phyris était considéré comme endémique du Nord-Est du Zaïre. Ceci est donc la première station connue en dehors de ce pays. Dans les deux cas, il apparaît comme orophile.

C'est apparemment une espèce éclectique. Alors que dans la région du Kivu (op. cit.) aucun hôte préférentiel ne se dégageait, ici 10 sur 12 proviennent de Lophuromys. Hypsophthalmus campestris J. et R. 1913.

Cette espèce est, apparemment, le seul représentant de cette famille présent avec une certaine fréquence dans cette région d'Afrique; tous les auteurs s'accordent cependant pour la considérer comme peu abondante (Hopkins, 1947 ; Smit, 1975 ; Beaucournu et Rahm, 1978). Sa répartition connue comprenait l'est du Zaïre, l'Ouganda, et pro parte le Kenya, ses hôtes étant des muridés, dont Lophuromys, et des musaraignes. Beaucournu et Rahm (op. cit.) avaient noté que, dans la région du lac Kivu, son biotope électif semblait être "en zone ouverte et humide à des altitudes de 1750 à $2000 \mathrm{~m} . .$. ».

C'est peut-être grâce au piégeage intensif fait dans un tel biotope que, à Tora, nous pouvons considérer $H$. campestris comme commune puisqu'elle représente $13 \%$ des captures (82 exemplaires), pourcentage étonnant pour une puce de ce genre.

Sa spécificité pour Lophuromys est confirmée (43\% viennent de cet hôte); deux autres rongeurs l'hébergent avec une certaine fréquence, Mastomys (22\%) et Oenomys $(20 \%)$.

Le sex-ratio d'H. campestris n'a jamais été établi. En ajoutant les données obtenues au Kivu par Beaucournu et Rahm (op. cit.) (12 mâles, 9 femelles) aux nôtres (43 mâles, 39 femelles) nous obtenons une fois encore un sex-ratio déséquilibré et anormal pour un siphonaptère $(1,14)$. Il est bien évident, toutefois que ces résultats demandent à être vérifiés par une meilleure approche du sex-ratio primaire, en étudiant la faune des nids et $a$ fortiori par l'élevage.

Xiphiopsylla hyparetes J. et R. 1913.

$X$. hyparetes est une espèce rare, connue du Kenya et de l'est du Zaïre. Ses hôtes sont malaisés à cerner, il s'agit peut-être du genre Lophuromys.

Nous en avons collecté 3 exemplaires à Tora ( 2 mâles, 1 femelle), 2 provenant de Lophuromys et 1 de Lemniscomys.

Xiphiopsylla levis Smit 1960.

Bien que ce soit l'une des dernières à avoir été isolée, $X$. levis, jamais abondante, est toutefois la plus fréquemment citée dans ce genre. Sa répartition connue touche 
le Kenya, l'Ouganda, l'est du Zaïre, et maintenant donc le Burundi. Elle est signalée d'un grand nombre de rongeurs muridés, cricétidés, rhizomyidés et même, accidentellement peut-être, de l'insectivore Chrysochloris. Lophuromys et Otomys renferment, sans doute, ses hôtes électifs.

Quatre femelles seulement ont été récoltées dans notre station : 3 sur Lophuromys et 1 sur Oenomys.

Nos piégeages ne portent que sur un demi-cycle annuel et il est impossible d'essayer d'en déduire une quelconque phénologie. Nous pouvons néanmoins noter que les 7 exemplaires de Xiphiopsylla collectés à Tora $(4 X$. levis et $3 X$. hyparetes $)$ l'ont été en avril. Il y a sans doute une relation avec le régime des pluies : à Tora, rappelons-le, la petite saison des pluies s'arrête à la mi-mai statistiquement; elle a été un peu plus précoce en 1978.

Nosopsyllus incisus (J. et R. 1913).

$N$. incisus est intéressant sur le plan paléogéographique en ce qu'il est l'unique représentant du genre, endémique dans la région éthiopienne. Sa morphologie le rattache nettement au complexe "fasciatus » originaire probablement d'Asie mineure.

La répartition connue de $N$. incisus comprend le sud de l'Ethiopie, le Kenya, l’Ouganda, le Zaïre oriental, la Tanzanie, le Malawi et la Zambie. Sa présence au Burundi est donc normale, mais on peut relever que cette vaste dispersion est sans doute lacunaire puisque cette espèce ne figure pas dans la faune récolté par Beaucournu et Rahm (1978) dans la région du lac Kivu.

Les hôtes de $N$. incisus sont des rongeurs: muridés essentiellement mais aussi cricetidés et muscardinidés.

A Tora, N. incisus est peu rare (41 captures), son hôte préférentiel étant ici Oenomys hypoxanthus avec près des $2 / 3$ des exemplaires.

Bien qu'on ne puisse juger sur des chiffres aussi faibles notons que nos Nosopsyllus se répartissent en 14 mâles et 27 femelles, soit pratiquement le double pour ces dernières.

Ctenocephalides felis strongylus (Jordan, 1925).

C. felis strongylus est une puce uniquement éthiopienne, ubiquiste et très peu spécifique. Présente partout, elle parasite des rongeurs, des lagomorphes, mais surtout divers ongulés et carnivores, sauvages ou domestiques, et l'homme. A propos de ce dernier, Pollitzer (1954) a pu écrire «Ctenocephalides felis strongylus remplace Pulex irritans au Congo belge et en Ouganda comme puce de l'homme " et cela a encore, assez récemment, été reconfirmé pour l'Ouganda par Krampitz (1968).

Sur les rongeurs de Tora, une seule capture en a été faite sur un hôte capturé au voisinage d'habitations. Il faut noter que cette puce y abonde cependant sur les chiens, dans des poulaillers, etc... Sur l'homme elle est présente, mais est manifestement concurrencée par Pulex irritans qui, dans nos relevés sur cet hôte, est actuellement la plus fréquente (Guiguen et Beaucournu, sous presse). Nous en reparlerons plus loin. 
Xenopsylla brasiliensis (Baker 1904) et X. cheopis (Rothschild 1903).

Ces deux puces sont largement répandues en Afrique et sont présentes partout, semble-t-il, où Rattus et Mastomys existent, mais il faut bien noter qu'il s'agit essentiellement de "faune synanthrope » les captures se faisant rares, voire absentes, en zone selvatique d'altitude telle celle que nous étudions ici.

Dans notre station, $X$. cheopis n'a été récolté que dans les huttes ou à leur voisinage sur Mastomys et Rattus. X. brasiliensis a été en revanche collecté sur un Oenomys selvatique.

Il est très important de noter à Tora l'occurrence, dans les huttes ou leur voisinage, de ces vecteurs de Yersinia pestis. Non pas qu'ils soient obligatoirement les plus valables, mais parce qu'ils constituent, non seulement le maillon entre la peste des rongeurs selvatiques et celle des rongeurs synanthropes, mais encore parce qu'éventuellement ces puces «domestiques» peuvent piquer l'homme.

\section{IV. - Discussion et Conclusion}

Cette première approche de la faune des Siphonaptères du Burundi montre l'importance que ces vecteurs risquent d'acquérir dans ce pays.

L'endémie de Typhus murin est déjà connue. Des observations de rongeurs sur l'un des foyers ont montré l'abondance de Rattus : 4 d'entre eux capturés ont donné un indice pulicidien de 4,75 , les puces notées étant toutes $X$. brasiliensis.

En ce qui concerne la Peste, en dépit de la proximité de foyers voisins anciennement connus et dont certains du Zaïre, de Tanzanie, ont des flambées régulières, il ne nous a pas été signalé d'épidémie au Burundi. Il nous paraît improbable que cette situation se pérennise. D'une part, le «nomadisme » se poursuit, voire s'accentue temporairement pour des raisons économiques ou politiques : or les bords du lac Tanganyika comme les rives de la Ruzizi, zones de passage privilégiées, forment l'un des côtés du Burundi. D'autre part, nous venons de découvrir à Tora, comme à Bujumbura la Capitale, stations prises au hasard, Pulex irritans, puce d'implantation récente en zone éthiopienne et qui peut bouleverser dans ces régions l'épidémiologie de la peste (Karimi et Fayrang-Azad, 1974).

Pour affermir malheureusement ces craintes, il convient de rappeler que dans cette station de Tora, qu'il nous faut bien prendre pour exemple, tous les éléments sont en place dans l'attente du bacille de Yersin: climatologie très comparable à celle des deux principaux foyers zaïrois (cf. en particulier Velimirovič et coll., 1969) ; forte densité de rongeurs sensibles; présence de rongeurs selvatiques, de rongeurs synanthropes et de rongeurs ambivalents (Mastomys); indice pulicidien élevé; présence de bons vecteurs à tous ces niveaux (Dinopsyllus spp. et Ctenophthalmus spp. pour le selvatique, Xenopsylla spp. pour le domestique ; Pulex au niveau humain) ; densité humaine élevée, enfin. Du fait de ce haut risque pesteux, nous pensons qu'il est important que des enquêtes plus systématiques, tant dans le domaine des rongeursréservoirs que dans celui des vecteurs, viennent compléter nos observations dans ce pays. 


\section{Bibliographie}

Beaucournu J.C., Rahm U. (1978) : Contribution à l'étude des Siphonaptères de rongeurs et d'insectivores dans la région occidentale du lac Kivu (Zaïre). Acta Tropica, 35, 357-372.

Davis D.H.S. (1953) : Plague in Africa from 1935 to 1949. A survey of wild rodents in African territories. Bull. O.M.S., 9, 665-700.

Delany M. J. (1975) : The rodents of Uganda. British Museum (Nat. Hist.), London, VII + 165 pp.

Gratz N. G., Arata A. A. (1975) : Problems associated with the control of rodents in tropical Africa. Bull. O.M.S., 52, 697-705.

Guiguen C., Beaucournu J.-C. (1979) : Présence de Pulex irritans (Siphonaptera) au Burundi, région à risque pesteux. Bull. Soc. Pathol. Exot. (sous presse).

Haeselbarth E. (1966) : Siphonaptera in Zumpt F.: The arthropod parasites of vertebrates in Africa South of the Sahara (Ethiopian Region), vol. III (Insecta excl. Phthiraptera). South African Institute for medical Research, Johannesburg, 283 p.

Hopkins G. H.E. (1947) : Annotated and illustrated keys to the known fleas of East Africa. Uganda J., Scientific suppl., 11, 138-190.

Karimi Y., Farhang-Azad A. (1974) : Sur Pulex irritans, puce humaine dans le foyer de la peste au lac du Général Mobutu (ancien lac Albert): déduction épidémiologique. Bull. O.M.S., 50, 564-565.

Krampitz H. E. (1968) : Felduntersuchungen zur Verbreitung der Flohfauna in einer Kulturlandschaft Ost-Ugandas mit besonderer Beachtung des Menschen. Z. Tropenmed. Parasit., 19, 297-305.

Misonne X. (1959) : Les rongeurs des foyers de peste congolais. Ann. Soc. belg. Med. trop., 4, 437. 493.

Pollitzer R. (1954) : La Peste. Monogr. O.M.S., $\mathrm{n}^{\circ}$ 22, 737.

Riel J. van, Mol G. (1939) : La peste dans le nord du Kivu. Ann. Soc. belg. Med. Trop., 19, 453-462.

Smit F.-G.-A.-M. (1960) : Mission zoologique de l'I.R.S.A.C. en Afrique orientale (P. Basilewsky et N. Leleup, 1957). LIV : Siphonaptera. Ann. Mus. Congo Tervuren, Zool., 88, 345-364.

Smit F.G.A.M. (1963): Species-groups in ctenophthalmus (Siphonaptera: Hystrichopsyllidae). Bull. Brit. Museum (Nat. Hist.) Entomology, 14, 105-152 (+ 58 pl.).

Smit F.G. A.M. (1975) : Siphonaptera from the Cherangani Hills in Kenya. Rev. Zool. afr., 89, 191-210.

Smit F. G. A.M. (1976) : Two new east African mole-rat fleas (Siphonaptera, Hystrichopsyllidae). Rev. Zool. afr., 90, 46-52.

Velimirovič B., Zikmund V., Herman J. (1969) : Plague in the lake Edwards Focus; the Democratic Republic of Congo, 1960-1966. Z. Tropenmed. Parasit., 20, 373-387. 